S. Cho

Nagoya Math. J.

Vol. 140 (1995), 167-176

\title{
A CONSTRUCTION OF PEAK FUNCTIONS ON LOCALLY CONVEX DOMAINS IN $\mathbf{C}^{n}$
}

\author{
SANGHYUN $\mathrm{CHO}^{\dagger}$
}

\section{Introduction}

Let $\Omega$ be a smoothly bounded pseudoconvex domain in $\mathbf{C}^{n}$ and let $A(\Omega)$ denote the functions holomorphic on $\Omega$ and continuous on $\bar{\Omega}$. A point $p \in b \Omega$ is a peak point if there is a function $f \in A(\Omega)$ such that $f(p)=1$, and $|f(z)|<1$ for $z \in \bar{\Omega}-\{p\}$. The existence of peaking functions is a qualitative converse of the maximum principle: if $f \in A(\Omega)$, then

$$
|f(z)| \leq \sup _{b \Omega}|f|, \quad z \in \Omega .
$$

When $\Omega$ is strictly pseudoconvex, the situation with regard to peak functions is fairly well understood, but in the weakly pseudoconvex case we know very little. If $\Omega \subset \subset \mathbf{C}^{2}$ is pseudoconvex and $b \Omega$ is of finite type, Bedford and Fornaess [1], showed that there is a peak function in $A(\Omega)$. This method also works for finite type domains in $\mathbf{C}^{n}$ where the Levi-form of $b \Omega$ has $(n-2)$-positive eigenvalues. We also mention the work of Bloom [2], Hakim and Sibony [10], and Range [16] on the existence of peak functions with additional smoothness up to the boundary of $\Omega$, i.e., in the various subclass of $A(\Omega)$.

Recently Fornaess and McNeal [9] proposed a new method to construct peak functions on finite type domains in $\mathbf{C}^{2}$ and on decoupled domains in $\mathbf{C}^{n}$. Their method depends on the solvability of $\bar{\partial}$-equation with $L^{\infty}$ or Hölder estimates of the domain, and on the estimates of the Bergman kernel on and off the diagonal near $p \in b \Omega$. Here we propose a method different from Fornaess and McNeal's. Namely, we construct a regular bumping family of pseudoconvex domains outside $V$, and use Bishop's $\frac{1}{4}-\frac{3}{4}$ method directly on bumped domains. This method can be applied for the domains where the precise estimates of the Bergman kernel function on and off the diagonal are known but the $L^{\infty}$ or Hölder estimates of the

Received October 27, 1994.

$\dagger$ Partially supported by Non directed research fund, K.R.F. 1994, and by GARC-KOSEF, 1994 
$\bar{\partial}$-equation is not known.

The precise estimates of the Bergman kernel functions near $p \in b \Omega$ are known for instance, finite type domains in $\mathbf{C}^{2}[4,11]$, decoupled domains in $\mathbf{C}^{n}$ [12], finite type domains in $\mathbf{C}^{n}$ with the Levi-form of $b \Omega(n-2)$-positive eigenvalues $[6,7]$. Also the existence of peaking functions are known for these domains. In this paper we will construct a peak function for locally convex finite type domains in $\mathbf{C}^{n}$. We will use McNeal's [15] estimates of the Bergman kernel functions for locally convex domains. We state our main theorem as follows:

THEOREM 1.1. Let $\Omega \subset \subset \mathbf{C}^{n}$ be a smoothly bounded pseudoconvex domain and let $b \Omega$ be of finite type. Suppose $p \in b \Omega$ and $\Omega$ is convex near $p$. For each small neighborhood $V$ of $p$, there is a peak function that peaks at $p$ and extends holomorphically up to $b \Omega \backslash V$.

We will prove Theorem 1.1 in Section 4.

\section{Smooth bumping families}

Let $\Omega$ be a smoothly bounded pseudoconvex domain in $\mathbf{C}^{n}$ with smooth defining function $r$ and let $0 \in b \Omega$. If $g: \mathbf{C} \rightarrow \mathbf{C}$ is any smooth function with $g(0)=0$, let $\nu(g)$ denote the order of vanishing of $g$ at 0 . For a vector valued $G=\left(g_{1}, \ldots\right.$, $\left.g_{n}\right)$, let $\nu(G)$ denote the minimum order of vanishing of the $g_{i}$ at 0 .

Definition 2.1 (D'Angelo). 0 is a point of finite 1 -type if

$$
\sup _{G} \frac{\nu\left(r^{\circ} G\right)}{\nu(G)}=\Delta(0)<\infty,
$$

where $G: \mathbf{C} \rightarrow \mathbf{C}^{n}$ is a complex analytic map with $G(0)=0 ; \Delta(0)$ is called the type of 0 .

Definition 2.2. Let $p \in b \Omega$ be an arbitrary point and let $V$ be a neighborhood of $p$. By a smooth bumping family for $\Omega$ outside $V$ we mean a family $\left\{\Omega_{t}\right\}_{0 \leq t \leq 1}$ of pseudoconvex domains with $\mathrm{C}^{\infty}$ defining functions $\left\{r_{t}\right\}$ with the following properties:

(a) $\Omega=\Omega_{0}$,

(b) $\Omega_{t_{1}} \subset \Omega_{t_{2}}$ if $t_{1}<t_{2}$, and $r_{t}(z)$ is smooth in $z$ and $t$,

(c) for any neighborhood $U$ of $\partial \Omega \backslash V$ there is a $t_{0}>0$ such that $\Omega_{t} \backslash U=$ $\Omega \backslash U$ for all $t \in\left[0, t_{0}\right]$.

The following theorem can be found in [5]. 
THEOREM 2.3. Let $p$ be a point of finite 1-type in the boundary of a pseudoconvex domain $\Omega$ in $\mathbf{C}^{n}$ with smooth defining function $r(z)$. Then for each neighborhood $V$ of $p$, there exists a smooth 1-parameter family of pseudoconvex domains $\left\{\Omega_{t}\right\}_{0 \leq t<t_{0}}$, each defined by $\Omega_{t}=\{z ; r(z, t)<0\}$, where $r(z, t)$ has the following properties:

(a) $r(z, t)$ is smooth in $z$ near $b \Omega$, and in $t$ for $0 \leq t<t_{0}$,

(b) $r(z, t)=r(z)$ for $z \notin V$,

(c) $\frac{\partial r}{\partial t}(z, t) \leq 0$,

(d) $r(z, 0)=r(z)$,

(e) for $z$ in $V, \frac{\partial r}{\partial t}<0$.

Definition 2.4. Suppose $\Omega, p \in b \Omega, V$ be as Theorem 2.3. Then we say $\left\{\Omega_{t}\right\}_{0 \leq t<t_{0}}$ a bumping family of $\Omega$ with front $V$.

THEOREM 2.5. Let $\Omega \subset \subset \mathbf{C}^{n}$ be a smoothly bounded pseudoconvex domain and let $b \Omega$ be of finite 1-type. Assume $p \in b \Omega$ and $V$ is a small neighborhood of $p$. Then there is a 1-parameter family of a smooth bumping family $\left\{\Omega_{t}\right\}$ outside $V$.

Proof. Choose a neighborhood $U$ of $p$ such that $V \subset \subset U$. Since $b \Omega$ is compact, we can choose points $z_{1}, \ldots, z_{N} \in b \Omega$ and $\varepsilon_{1}, \ldots, \varepsilon_{N}>0$ such that

(1) $\Omega$ is pseudoconvex and $b \Omega$ is of finite type,

(2) $\cup_{i=1}^{N} B\left(z_{i}, \varepsilon_{i} / 2\right) \supset b \Omega \backslash U$,

(3) $V \cap B\left(z_{i}, \varepsilon_{\imath}\right)=\varnothing, i=1,2, \ldots, N$,

(4) $\overline{B\left(z_{i}, \varepsilon_{i}\right)}$, is contained in a neighborhood $V_{i}$ where Theorem 2.3 can be applicable, $i=1,2, \ldots, N$.

Set $V_{i}=B\left(z_{i}, \varepsilon_{i}\right), i=1,2, \ldots, N$, for the convenience. Consider a bumping family of $\Omega$ with front $V_{1}$. Since the type condition is stable under small $C^{\infty}$-perturbations of $b \Omega$, we will get a family $\left\{\Omega_{t_{1}}\right\}_{0 \leq t_{1}<\alpha_{1}}$ of smooth pseudoconvex domains satisfying (1)-(4) for the domains $\Omega_{t_{1}}$ (instead of $\Omega$ ) provided $\alpha_{1}$ is sufficiently small. For each $\Omega_{t_{1}}, 0 \leq t_{1}<\alpha_{1}$, we consider a bumping family of $\Omega_{t_{1}}$ with front $V_{2}$ and call it $\left\{\Omega_{t_{1} t_{2}}\right\}_{0 \leq t_{2}<\alpha_{2}}$. Again $\left\{\Omega_{t_{1} t_{2}}\right\}_{0 \leq t_{2}<\alpha_{2}}$ will satisfy (1)-(4) provided $\alpha_{2}$ is sufficiently small. Continuing in this manner, we will get a bumping family of pseudoconvex domains $\left\{\Omega_{t_{1} t_{2} \ldots t_{N}}\right\}$ outside $V$. Obviously we can regard this family as a 1-parameter family of pseudoconvex domains.

\section{Estimates on the Bergman kernel}

In this section, we estimate the Bergman kernel function on a locally convex 
domains in $\mathbf{C}^{n}$. For the estimates of the kernel on the other domains, one can refer $[4,6,7,11,12]$.

Let $\Omega \subset \subset \mathbf{C}^{n}$ be smoothly bounded and convex in some neighborhood $U$ of $p \in b \Omega$. Suppose that $p$ is a point of finite type $T$ in the sense of D'Angelo [8]. In [13], McNeal showed that $T$ is actually the maximum order of contact of $b \Omega$ with complex lines at $p$. The convexity of $b \Omega$ in $U$ is independent of the choice of the local defining function. Furthermore we may assume that the defining function $r$ for $U \cap b \Omega$ has the property that all the sets $\{z ; r(z)<\eta\} \cap U$ are convex for $\eta$ in some range $-\eta_{0}<\eta<\eta_{0}, \eta_{0}>0$. Let $S^{n}=\left\{\zeta \in \mathbf{C}^{n} ;|\zeta|=1\right\}$. Then each element of $S^{n}$, together with the point $q$ near $p$ determines a complex line in $\mathbf{C}^{n}$. If $\zeta \in S^{n}$ and $\eta_{0}<\eta<\eta_{0}$, we denote the distance from $q$ to the level set $\{z ; r(z)$ $=\eta\}$ along the complex line determined by $\zeta$ by $\delta_{\eta}(q, \zeta)$. Assume $p=0 \in b \Omega$.

Proposition 3.1 (15, Proposition 2.1). After perhaps shrinking $U$, for every $q \in$ $\Omega \cap U$ and every $\varepsilon>0$ sufficiently close to 0 , there exist coordinates $\left(z_{1}, \ldots, z_{n}\right)$ centered at $q$, positive numbers $\tau_{1}(q, \varepsilon), \ldots, \tau_{n}(q, \varepsilon)$, and points $p_{1}, \ldots, p_{n} \in\{z ; r(z)$

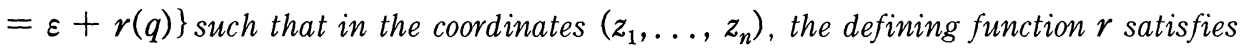

(i) for $1 \leq i \leq n$,

$$
\frac{\tau_{1}(q, \varepsilon)}{\tau_{i}(q, \varepsilon)} \leqslant \frac{\partial r}{\partial z_{i}}\left(p_{i}\right) \mid \leqslant \frac{\tau_{1}(q, \varepsilon)}{\tau_{i}(q, \varepsilon)},
$$

(ii) if $i<j$,

$$
\left|\frac{\partial r}{\partial z_{i}}\left(p_{i}\right)\right| \lesssim \frac{\tau_{1}(q, \varepsilon)}{\tau_{i}(q, \varepsilon)}
$$

(iii) if $i>j, \quad\left|\frac{\partial r}{\partial z_{i}}\left(p_{i}\right)\right|=0$.

Also if we define the polydisc

$$
P_{\varepsilon}(q)=\left\{z \in U ;\left|z_{1}\right|<\tau_{1}(q, \varepsilon), \ldots,\left|z_{n}\right|<\tau_{n}(q, \varepsilon)\right\},
$$

then there exists a constant $c>0$, independent of $q \in \Omega \cap U$, such that $c P_{\varepsilon}(q) \subset\{z$ $\in U ; r(z)<\varepsilon+r(q)\}$.

Let us introduce a quantitative estimate on the weights $\tau_{i}(q, \varepsilon)$. Let $z_{j}=x_{j}$ $+i x_{j+n}$ for $1 \leq j \leq n$ denote the underlying real coordinates. For each $2 \leq i \leq n$, an application of Taylor's theorem gives

$$
\begin{aligned}
r \circ z\left(0, \ldots, x_{i}, 0 \ldots, 0\right) & =r(q)+\sum_{k=2}^{T} a_{k}^{i}(q) x_{i}^{k}+\mathscr{O}\left(\left|x_{i}\right|^{T+1}\right) \\
& =r(q)+f_{i}\left(x_{i}\right) .
\end{aligned}
$$


Since each $\tau_{i}(q, \varepsilon)$ is the maximum distance in $\operatorname{Re} z_{j}$ direction to the level surface $\{z ; r(z)=r(q)+\varepsilon\}$, we have that

$$
\varepsilon+r(q)=r(q)+\sum_{k=2}^{T} a_{k}^{i}(q) \tau_{\imath}(q, \varepsilon)^{k}+\mathscr{O}\left(\left|\tau_{\imath}(q, \varepsilon)\right|^{T+1}\right) .
$$

For $2 \leq k \leq T$, define

$$
A_{k}^{i}(q)=\left|a_{k}^{i}(q)\right|
$$

and for $\varepsilon>0$, set

$$
\sigma_{i}(q, \varepsilon)=\min \left\{\left(\varepsilon / A_{k}^{i}(q)\right)^{\frac{1}{q}} ; 2 \leq k \leq T\right\} .
$$

Then it was shown in [15] that, for each $2 \leq i \leq n$,

$$
\sigma_{i}(q, \varepsilon) \lesssim \tau_{i}(q, \varepsilon) \lesssim \sigma_{i}(q, \varepsilon) .
$$

From the construction in Proposition 3.1, $\tau_{1}(q, \varepsilon)$ is the distance from $q$ to $b D_{q, \varepsilon}$ where $b D_{q, \varepsilon}=\{z \in U ; r(z)=\varepsilon+r(q)\}$. Hence $\operatorname{Re} z_{1}$ is the normal direction and $\tau_{1}(q, \varepsilon) \approx \varepsilon$ independent of $q \in U$.

In these setting, McNeal got the following estimates for the Bergman kernel function.

THEOREM 3.2. Suppose $\Omega \subset \subset \mathbf{C}^{n}$ is smoothly bounded and pseudoconvex. Let $p \in b \Omega$ be a point of finite type $T$ and assume there is some neighborhood $U$ of $p$ so that $\Omega$ is convex in $U$. There exists a neighborhood $V \subset \subset U$ so that if $q \in V \cap \Omega$,

$$
K_{\Omega}(q, q) \approx \prod_{i=1}^{n} \tau_{i}(q, \delta)^{-2}
$$

where $\delta=|r(q)|$.

Suppose that $q^{1}, q^{2} \in U \cap \Omega$. Define

$$
M\left(q^{1}, q^{2}\right)=\inf \left\{\varepsilon>0 ; q^{2} \in P_{\varepsilon}\left(q^{1}\right)\right\},
$$

where $P_{\varepsilon}\left(q^{1}\right)$ is constructed from the coordinates about $q^{1}$ as in Proposition 3.1 . Set $\delta=M\left(q^{1}, q^{2}\right)$. Then

$$
M\left(q^{1}, q^{2}\right) \approx\left|q_{1}^{1}-q_{1}^{2}\right|+\sum_{i=2}^{n} \sum_{l=2}^{T} A_{l}^{i}\left(q^{1}\right)\left|q_{i}^{1}-q_{i}^{2}\right|^{l}
$$

where the coordinates of $q^{1}$ and $q^{2}$ are measured in the coordinates associated to $q^{1}$ and $\delta$ by Proposition 3.1. In the following, we let $D_{i}$ denote the differential operator $\frac{\partial}{\partial z_{i}}$ where $z_{i}$ is one of the coordinates constructed in Proposition 3.1 
associated to $q^{1}$. For multi-indices $\alpha$ and $\beta, D^{\alpha} \bar{D}^{\beta}=D_{1}^{\alpha_{1}} \ldots D_{n}^{\alpha_{n}} \bar{D}_{1}^{\beta_{1}} \ldots \bar{D}_{n}^{\beta_{n}}$ with the convention that the holomorphic derivatives act on the first $n$ variables of the kernel function and the anti-holomorphic derivatives act on the last $n$-variables.

THEOREM 3.3. Let $\Omega \subset \subset \mathbf{C}^{n}$ be a smoothly bounded, pseudoconvex domain. Suppose that near $p \in b \Omega, \Omega$ is convex and that $p$ is a point of finite type $T$. There exists a neighborhood $U$ of $p$ so that, for all multi-indices $\alpha, \beta$, there exists a constant $C_{\alpha \beta}$ such that for all $q^{1}, q^{2} \in U \cap \Omega$,

$$
\left|D^{\alpha} \bar{D}^{\beta} K_{\Omega}\left(q^{1}, q^{2}\right)\right| \leq C_{\alpha \beta} \prod_{i=1}^{n} \tau_{i}\left(q^{1}, \delta\right)^{-2-\alpha_{i}-\beta_{i}},
$$

where $\delta=\left(\left|r\left(q^{1}\right)\right|+\left|r\left(q^{2}\right)\right|+M\left(q^{1}, q^{2}\right)\right)$.

Lemma 3.4. Let $\Omega$ and $p \in b \Omega$ be as in Theorem 3.3. Then there exist a neighborhood $U$ of $p$ and a constant $C$ so that

$$
|K(z, q) / K(q, q)| \leq C
$$

for all $z, q \in U$.

Proof. Take $\alpha=\beta=0$ in (3.4). Then we have

$$
\left|K_{\Omega}(z, q)\right| \leq C^{\prime} \prod_{i=1}^{n} \tau_{i}(q, \delta)^{-2}
$$

where $\delta_{1}=\left(|r(z)|+|r(q)|+M\left(q^{1}, q^{2}\right)\right)$. Also from (3.3) we have $K_{\Omega}(q, q) \approx$ $\Pi_{i=1}^{n} \tau_{i}\left(q, \delta_{2}\right)^{-2}$, for $\delta_{2}=|r(q)|$. From (3.1), (3.2) and from the definition of $\tau_{i}$, one can see that $\tau_{i}(q, \delta)$ is an increasing function for $\delta$. So $\left|K_{\Omega}(z, q) / K_{\Omega}(q, q)\right|$ $\leq C$ for some $C>0$.

\section{Construction of peak functions}

Suppose that $\Omega \subset \subset \mathbf{C}^{n}$ is a smoothly bounded pseudoconvex domain and $p \in$ $b \Omega$ is a point of finite 1 -type. Then Catlin's theorem [3] says that there are $\varepsilon>0$ and a neighborhood $U$ in which $\bar{\partial}$-Neumann problem satisfies a subelliptic estimate of order $\varepsilon>0$ on $(0,1)$-forms. For each $w \in U \subset \Omega$, define the function

$$
h_{w}(z)=\frac{K_{\Omega}(z, w)}{K_{\Omega}(w, w)} .
$$

We will estimate $K_{\Omega}(z, w)$ for $z$ outside a certain neighborhood of $w$ and will 
show that $\left|h_{w}(z)\right|$ is quite small outside that neighborhood. For the convenience in notation, we denote by $C$ the various constants that follows.

Let $\pi$ be a projection onto $b \Omega$ and set $p=\pi(w)$. For $\eta>0$, let $B(p, \eta)$ denote the euclidean ball centered at $p$ of radius $\eta$. Let $\zeta \in C^{\infty}\left(\mathbf{C}^{n}\right)$ be a function with the property that $\zeta \equiv 1$ on $\Omega \backslash B(p, \eta)$ and $\zeta \equiv 0$ on $B\left(p, \frac{\eta}{2}\right)$, and let $N$ denote the $\bar{\partial}$-Neumann operator on $(0,1)$-forms. The following theorem was proved in [14].

Proposition 4.1. Let $\Omega, U$, and $\varepsilon>0$ be as above. Let $s, t \in \mathbf{R}^{+}$. If $\alpha$ is a smooth $(0,1)$-form in the domain of the Kohn Laplacian and $\operatorname{supp} \alpha \subset B\left(p, \frac{\eta}{8}\right)$ then there is a constant $C_{s t}>0$ so that

$$
\|\zeta N \alpha\|_{s}^{2} \leq C_{s t} \eta^{-2\left(\frac{s+t}{\varepsilon}+4\right)}\|\alpha\|_{-t}^{2} .
$$

Let $\phi \in C_{0}^{\infty}(0,1)$ be a non-negative radial function with $\int \phi=1$. For $w \in$ $U$, set

$$
\phi_{w}(z)=\left(\frac{\delta(w)}{2}\right)^{-2 n} \phi\left(\frac{z-w}{\delta(w) / 2}\right)
$$

Then from the Kohn's formula,

$$
K_{\Omega}(z, w)=\phi_{w}(z)-\bar{\partial}^{*} N \bar{\partial} \phi_{w}(z) .
$$

Assume $\operatorname{supp} \phi_{w} \subset B\left(p, \frac{\eta}{8}\right)$. Then from the Proposition 4.1 with $s=r+1$, we have

$$
\begin{aligned}
\left\|\zeta(\cdot) K_{\Omega}(\cdot, w)\right\|_{r}^{2} & \leq C\left\|\zeta N \bar{\partial} \phi_{w}(\cdot)\right\|_{r+1}^{2} \\
& \leq C \eta^{-2\left(\frac{r+1+t}{\varepsilon}+4\right)}\left\|\bar{\partial} \phi_{w}(\cdot)\right\|_{-t}^{2} .
\end{aligned}
$$

If $t>n+1$, Sobolev's lemma gives

$$
\begin{aligned}
\left\|\phi_{w}\right\|_{-(t-1)}^{2} & =\sup \left\{\left|\left(\phi_{w}, f\right)\right| ; f \in C_{0}^{\infty}, \quad\|f\|_{t-1} \leq 1\right\} \\
& \leq C\left|\int \phi_{w}\right| \leq C
\end{aligned}
$$

for some $C>0$. If we choose $r>n+1$, another application of Sobolev's lemma shows

$$
\sup _{z}\left|\zeta(z) K_{\Omega}(z, w)\right| \leq C\left\|\zeta(\cdot) K_{\Omega}(\cdot, w)\right\|_{r}
$$


Hence

$$
\sup _{z}\left|\zeta(z) K_{\Omega}(z, w)\right| \leq C \eta^{-2\left(\frac{2 n+4}{\varepsilon}+4\right)} .
$$

Now set $\eta=\delta(w) \frac{\varepsilon}{\left(\frac{\varepsilon}{2(2 n+4)}\right)}$. If we combine Theorem 3.2 and Theorem 3.3, we have proved the following:

Proposition 4.2. Let $\Omega$ and $\eta$ be as above. There exists a constant $C>0$, independent of $w \in U$, so that

$$
\left|h_{w}(z)\right| \leq C \delta(w)
$$

for $w \in U$ and $z \in \bar{\Omega} \backslash B(\pi(w), \eta)$.

Now we denote by $N$ the interior normal to the boundary of $\Omega$ at $p$. In [15], McNeal showed that the sharp subelliptic estimates of $\bar{\partial}$-equation holds near $p \in$ $b \Omega$. So we may take $\varepsilon=\frac{1}{T}$. Set $\eta=\frac{1}{2(2 n+4) T}$.

Lemma 4.3. For every $q$ on $N$, let $|q-p|=d$. There exists a constant $C>0$ such that for every point $q$ on $N$ sufficiently close to $p$, there exist a neighborhood $U_{p}$ of $p$ and a holomorphic function $h=h_{q}$ on $\Omega$ such that

(1) $|h|<C$ on $\Omega$,

(2) $h(q)=1$,

(3) $|h(z)|<C d$ for $z \in \Omega \backslash U_{p}$,

(4) $|D h|<\frac{C}{d}$.

Proof. Define $h_{q}(z)=K(z, q) / K(q, q)$. Property (2) is clear. From Proposition 4.2 , we have $\left|h_{q}(z)\right| \leq C d$ for $z \in \bar{\Omega} \backslash B\left(\pi(q), d^{\eta}\right)$. This proves (3) with $U_{p}=B\left(p, d^{\eta}\right)$. Lemma 3.4 gives $|h| \leq C$ for $z \in U_{p}=B\left(\pi(q), d^{\eta}\right)$. This fact together (3) gives (1). Also from the estimated of Theorem 3.2 and Theorem 3.3, we have $|D h|<\frac{C}{d}$.

We now ready to prove Theorem 1.1 .

Proof of Theorem 1.1. We may assume that $p=0$. Let the type of $p$ is equal to $T$ and choose a neighborhood $U$ of $p$ such that the subelliptic estimates for $\bar{\partial}$-equation of order $\frac{1}{T}$ hold on $U$ and Proposition 3.1, Theorem 3.2 and Theorem 
3.3 hold on $U$. We denote by $N$ the interior normal to the boundary of $\Omega$ at $p$. For each neighborhood $V \subset \subset U$ of $p$, choose a neighborhood $V_{1}, V_{1} \subset \subset \subset \subset U$, so that $V_{1} \cap \Omega$ is convex. Next we consider a 1 -parameter family of a smooth bumping family $\left\{\Omega_{t}\right\}_{0 \leq t<t_{0}}$ outside $V_{1}$. We may assume that $V_{1} \cap \Omega=V_{1} \cap \Omega_{t}$ for all $t>0$, after perhaps shrinking $V_{1}$, and $\Omega \backslash V \subset \subset \Omega_{t} \backslash V$ for all $0<t<t_{0}$. Now fix $0<t_{1}<t_{0}$ and consider the pseudoconvex domain $\Omega_{t_{1}}$. Since the type condition is stable, we may assume that $b \Omega_{t_{1}}$ is of finite type. Also we have $d=$ $\operatorname{dist}\left(\Omega \backslash V, b \Omega_{t_{1}} \backslash V\right)>0$. Choose (by induction) a sequence $q_{n}$ coverging to $p$ along $N$ and define $h_{n}(z)=h_{q_{n}}\left(z+q_{n}-p\right)$, where $h_{q_{n}}$ is the function defined on $\Omega_{t_{1}}$ (instead of $\Omega$ ) as in Lemma 4.3 associated with $q_{n}$. Notice that $h_{n}(z)$ is defined on $\bar{\Omega} \backslash V$, and on $U$ intersect a translate of $\Omega$ which contains $\bar{\Omega} \cap \bar{V}$, provided $q_{0}$ is sufficiently close to $p$. Therefore $h_{n}$ is well defined and holomorphic on $\bar{\Omega}$ for each $n \geq 0$. Let $U_{n}$ denote the neighborhood corresponding to $h_{n}$ as in Lemma 4.3. Without loss of generality, we may assume that $U_{n+1} \subset \subset U_{n}$. For a suitable constant $0<c<1$, to be determined later, let $r=1-c$ and define a peak function as $H=r \sum_{n=0}^{\infty} c^{n} h_{n}$. Let us estimate $H$ on various sets. First outside $U_{0}$. Then $\left|h_{n}\right|<\frac{1}{2}$ for every $n$ by the property (3) of Lemma 4.3 provided that $q_{0}$ is sufficiently close to $p$. So we get that $|H|<r \sum_{n=0}^{\infty} \frac{c^{n}}{2}=\frac{1}{2}$. From the continuity of $h_{k}, 0 \leq k \leq n-1$, and from the fact that $h_{k}(p)=1$, we may (inductively) choose $q_{n}$ and hence a neighborhood $U_{n}$ so that $\left|h_{k}\right|<r_{n}$ for $k<n$ on $U_{n}$, where $r_{n}>1$ is arbitrary close to 1 . Hence for $z \in U_{n} \backslash U_{n+1}$, we have that $\left|h_{n}\right|<C$ and $\left|h_{k}(z)\right|<\frac{1}{2}$ (by the property (3) of the Lemma 4.3) if $k>n$. Hence for $z \in$ $U_{n} \backslash U_{n+1}$, we estimate $H$ as follows

$$
\begin{aligned}
|H| & <r\left[\sum_{k<n} r_{n} c^{k}+c^{n} C+\sum_{k>n} c^{k} 2\right] \\
& =r \frac{\left(1-c^{n}\right)}{1-c} r_{n}+r c^{n} C+\frac{r}{2} \frac{c^{n+1}}{2(1-c)} \\
& =\left(1-c^{n}\right) r_{n}+\frac{1}{2} c^{n+1}+r C c^{n} \\
& =1-c^{n} s_{n}+\frac{1}{2} c^{n+1}+r c^{n} C
\end{aligned}
$$

where $s_{n}<1$ can be chosen arbitrary close to 1 . If $r C=\frac{1}{3}$, for instance, we have $|H|<1$ and $r=\frac{1}{3 C}, c=\frac{1}{3 C}$. Notice that $H(p)=1$ and $H$ is continuous on $\bar{\Omega}$ 
and holomorphic on $\bar{\Omega} \backslash V$ and this proves Theorem 1.1 .

Remark 4.4. In the proof of Theorem 1.1, we may take, for example, $r_{n}=1+$ $\frac{1}{10} c^{n}$ and $s_{n}=1-\frac{1}{10}+\frac{c^{n}}{10}$.

\section{REFERENCES}

[1] Bedford, E. and Fornaess, J. E., A construction of peak functions on weakly pseudoconvex domains, Ann. of Math., 107 (1978), 555-568.

[2] Bloom, T., $C^{\infty}$ peak functions for pseudoconvex domains of strict type, Duke Math. J., 45 (1978), 133-147.

[ 3 ] Catlin, D. W., Subelliptic estimates for the $\bar{\partial}$-Neumann problem on pseudoconvex domains, Ann. of Math., 126 (1987), 131-191.

[4] Catlin, D. W., Estimates of invariant metrics on pseudoconvex domains of dimension two, Math. Z., 200 (1980), 429-466.

[5] Cho, S., Extension of complex structures on weakly pseudoconvex compact complex manifolds with boundary, Math. Z., 211 (1992), 105-120.

[6] Cho, S., Boundary behavior of the Bergman kernel function on some pseudoconvex domains in $\mathbf{C}^{n}$, Trans. Amer. Math. Soc., 345 (No.2) (1994), 803-817.

[7] Cho, S., Estimates of the Bergman kernel function on certain pseudoconvex domains in $\mathbf{C}^{n}$, Math. Z., to appear.

[ 8 ] D'Angelo, J., Real hypersurfaces, order of contact, and applications, Ann. of Math., 115 (1982), 615-637.

[9] Fornaess, J. E. and McNeal, J., A construction of peak functions on some finite type domains, Amer. J. Math., 116 (No.3) (1994), 737-755.

[10] Hakim, M. and Sibony, N., Quelques conditions pour l'existence de fonctions.pics dans des domaines pseudoconvexes, Duke Math. J., 44 (1977), 399-406.

[11] McNeal, J., Boundary behavior of the Bergman kernel functions in $\mathbf{C}^{2}$, Duke Math. J., 58 No.2 (1989), 499-512.

[12] McNeal, J., Local geometry of Decoupled pseudoconvex domain, Proc. in honor of Grauert, H., Aspekte der Math., Vieweg, Berlin (1990), 223-230.

[13] McNeal, J., Convex domains of finite type, J. Funct. Anal., 108 (No.2) (1992), $361-373$.

[14] McNeal, J., Lower bounds on the Bergman metric near a point of finite type, Ann. of Math., 136 (1992), 361-373.

[15] McNeal, J., Estimates on the Bergman kernels of convex domains, Adv. in Math. (to appear).

[16] Range, R. M., The Caratheodory metric and holomorphic maps on a class of weakly pseudoconvex domains, Pacific J. Math., 78 (1978), 173-188.

Department of Mathematics Edu.

Pusan University

Pusan 609-735, Korea

E-mail: cho@hyowon.cc.pusan.ac.kr 